\title{
Kompressionsverband kombiniert Vorteile von Kurz- und Langzugbinde
}

\begin{abstract}
- Der Zweilagen-Kompressionsverband UrgoK $2^{\circledast}$ gewährleistet einen anhaltend hohen Anpressdruck von über $40 \mathrm{mmHg}$ über sieben Tage und reduziert bei gutem Tragekomfort das Beinvolumen stärker als vergleichbare Mehrlagen-Verbände. Das zeigt eine Studie von Jünger und Mitarbeitern, die den Zweilagen- mit einem Vierlagen-Verband und einer kohäsiven Kurzzugbinde an 24gesunden Freiwilligen verglichen (sieben Männer, 17 Frauen). Untersucht wurden jeweils zwölf Beine pro Verbandsgruppe. Eine spezielle Sonde maß den Anpressdruck im Liegen, Sitzen, Stehen und in Bewegung (Arbeitsdruck).
\end{abstract}

Bei allen drei Verbänden hielt sich der therapeutisch notwendige Druck über sieben Tage über $40 \mathrm{mmHg}$, der Druckabfall war in allen Gruppen akzeptabel und lag für den Zweilagen-Verband bei etwa 20\%. Auch sorgten alle drei Verbände für eine Volumenreduktion des Unterschenkels, wie Studienleiter Prof. Dr. med. Michael Jünger aus Greifswald erklärte: „Mit UrgoK $2{ }^{\circledR}$ wurde das Volumen jedoch am ausgeprägtesten vermindert." Günstiger schnitt der Zweilagen-Verband auch hinsichtlich unerwünschter Nebenwirkungen und Tragekomfort ab: Schmerzen, Brennen, Schwitzen und Juckreiz traten seltener auf; die Passgenauigkeit war besser;
Austrocknung der Haut, Verrutschen sowie Beschwerden im Sitzen und Gehen waren geringer ausgeprägt.

Erreicht werden diese Effekte durch die Kombination von Kurz- und Langzugbinde. Die innere Kurzzugbinde verteilt den Druck gleichmäßig, und absorbiert Wundexsudat. Die äußere elastische kohäsive Langzugbinde baut weiteren Druck auf und sorgt für die nötige Formstabilität. Spezielle Markierungen auf den Binden zeigen die korrekte Zugstärke an.

koc

Symposium „Kompression und Wundversorgung bei Ulcus cruris venosum: Neue Behandlungsoption mit UrgoK $2^{\circledR}$ und UrgoCell ${ }^{\circledR}$ Contact", im Rahmen der 12. Tagung der DGfW. Kassel, 26.6.2009 (Veranstalter: Urgo $\mathrm{GmbH}$ ).

\section{Behandlung der schweren Psoriasis}

- Kürzlich wurden die Zweijahresdaten der REVEAL-Studie vorgestellt: Hier zeigte sich. Auch langfristig ist die Therapie mit Adalimumab (Humira ${ }^{\circledR}$ ) sicher und effektiv.

Die Studie „Randomized Controlled Evaluation of Adalimumab Every Other Week Dosing in Moderate to Severe Psoriasis TriAL (REVEAL)“ untersuchte die kurz- und langfristige Wirksamkeit und Sicherheit von Adalimumab sowie das Risiko eines Remissionsverlusts nach Absetzen des Medikaments an über 1200 Patienten. Nach der eigentlichen einjährigen Studienphase wurde die Untersuchung ein Jahr lang offen fortgesetzt. Die jetzt vorgestellten Ergebnisse dieser Verlängerung zeigen: $86 \%$ der ursprünglichen PASI-75-Responder wiesen auch nach zweijähriger Therapie mit dem Antikörper noch ein PASI-75-Ansprechen oder höher auf. Zu Beginn der Verlängerungsphase traf dies für $87 \%$ der Patienten zu, d.h. im zweiten Behandlungsjahr verringerte sich das PASI-75-Ansprechen um lediglich ein Prozent.

Bereits am Ende der eigentlichen Studienphase erlebten 28,4\% der Patienten, bei denen die Behandlung mit Adalimumab nach acht Monaten abgesetzt worden war, einen Verlust der Remission, bei den Patienten unter fortgesetzter Therapie mit Adalimumab waren es nur 4,9\%. Dies spricht dafür, die Therapie ausreichend lang durchzuführen.
Die Therapie mit dem Antikörper wird jetzt mit dem Pen bequemer und einfacher für die Patienten. Dies zeigte eine Studie, in der die Anwendung eines Humira-Pens im Vergleich zu Fertigspritzen untersucht wurde. Neun von zehn Patienten gaben an, dass die Handhabung des Pens einfacher sei und zudem weniger Schmerzen verursache. Daten der Abbott-Qualitätskontrolle zeigen, dass diese Anwendung in 99,8\% der Fälle erfolgreich ist.

kam

Pressegespräch „Management der Psoriasis 2009-Herausforderungen in der Praxis“, im Rahmen der 18. EADV-Tagung. Berlin, 9.10.2009 (Veranstalter: Abbott).

\section{Evidenzgestützte Babypflege}

Erste evidenzbasierte Pflegeempfehlungen bieten ab sofort Orientierung bei der Hautpflege von Neugeborenen und Säuglingen. Als Basis dienen unter anderem neueste Studien der Hautklinik der Charité, Berlin. und Empfehlungen eines europäischen Experten-Roundtables. Die Wichtigsten Empfehlungen lauten: — Babys sollten zwei- bis dreimal pro Woche gebadet werden.

_ Die Raumtemperatur sollte über 22 Grad Celsius betragen, die Wassertemperatur 37 bis 38 Grad Celsius.

_-Das Bad sollte nicht länger als fünf bis zehn Minuten dauern, das Kind anschließend sanft, aber rasch abgetrocknet werden.
_ Ein milder Babybadezusatz eignet sich für die schonende Reinigung besser als klares Wasser.

_ Der Badezusatz sollte speziell für Babyhaut entwickelt und flüssig sein (keine Seife).

_Es wird empfohlen, Säuglinge nach dem Baden mit einer Babypflegecreme einzucremen.

In einem Jahr soll an der Charité eine Evaluation stattfinden, wie sich das Konzept in der Praxis bewährt.

Nach Presseinformationen von Johnson \& Johnson 